\title{
Expression of cell adhesion molecule 1 in malignant pleural mesothelioma as a cause of efficient adhesion and growth on mesothelium
}

\author{
Akihiko Ito ${ }^{1,4}$, Man Hagiyama ${ }^{1,4}$, Takeshi Mimura², Masaki Matsumoto ${ }^{1}$, Tomohiko Wakayama ${ }^{3}$, Shoichi Iseki ${ }^{3}$, \\ Hiroshi Yokozaki ${ }^{1}$ and Morihito Okada ${ }^{2}$
}

Cell adhesion molecule 1 (CADM1), formerly referred to as SgIGSF, TSLC1, or Necl-2, has been characterized as a mast-cell adhesion molecule that mediates efficient interactions with mesothelial cells. Here, we examined whether CADM1 might be involved in the diffuse tumor growth over the pleural surface that characterizes malignant pleural mesothelioma (MPM). Immunohistochemical and western blot analyses revealed that 14 (25\%) of 57 MPMs expressed the full-length form of CADM1 on the cell membrane, but non-neoplastic mesothelial cells did not express it at all. The majority of available MPM cell lines also expressed the full-length form of CADM1. We compared CADM1-positive and -negative MPM cells in culture within soft agar and in coculture on mesothelial or fibroblastic monolayers. Within soft agar, CADM1-negative MPM cells were capable of forming colonies, whereas CADM1-positive cells were not, suggesting that CADM1 is a potential tumor suppressor of MPM, consistent with the past characterization of this molecule in other types of tumors. However, in coculture on mesothelial cell monolayers lacking full-length CADM1, CADM1-positive MPM cells spread more widely and grew more quickly, whereas the CADM1-negative cells piled up. Transfection of the CADM1-negative cells with CADM1 CDNA caused them to behave like the CADM1-positive cells, with faster, more widespread growth. These phenotypic differences were not detectable in cocultures on lung fibroblastic monolayers, in which all MPM cells grew much more slowly than on mesothelial cells, irrespective of CADM1 positivity. CADM1 thus appears to mediate efficient adhesion and growth of MPM cells specifically on mesothelial cells, probably via trans-heterophilic binding, and thus may be involved in the manifestation of a considerable subset of MPMs as diffusely growing tumors disseminated over the pleural surface.

Laboratory Investigation (2008) 88, 504-514; doi:10.1038/labinvest.2008.15; published online 10 March 2008

KEYWORDS: Necl-2; pleural dissemination; SgIGSF; TSLC-1

Lung cancers generally develop in the lung parenchyma and occasionally disseminate over the pleural surface as a result of progressive pleural involvement. Malignant pleural mesotheliomas (MPMs), however, are diffuse tumors that primarily grow on the pleural surface. ${ }^{1}$ Typically, early in the disease the tumor appears as multiple small nodules scattered over the pleura. As the disease progresses, the tumor nodules grow into plaque-like masses and finally become large confluent sheets that form a rind completely encasing the lung and heart. In contrast to this severe involvement of the parietal and visceral pleura, severe infiltration deep into the lung parenchyma is unusual even in advanced MPMs. Therefore, MPM cells seem to have a particular preference for interacting with mesothelial cells. Although this characteristic pattern of tumor growth is one of the major reasons this disease often has a poor prognosis, ${ }^{2}$ little attention has been paid to the molecular basis of MPM-mesothelial cell interaction. $^{3}$

\footnotetext{
'Division of Pathology, Graduate School of Medicine, Kobe University, Kobe, Japan; ${ }^{2}$ Department of Surgical Oncology, Research Institute for Radiation Biology and Medicine, Hiroshima University, Hiroshima, Japan and ${ }^{3}$ Department of Histology and Embryology, Graduate School of Medical Science, Kanazawa University, Kanazawa, Japan

Correspondence: Dr A Ito, MD, PhD, Division of Molecular Pathology, Department of Cancer Biology, Institute of Medical Science, The University of Tokyo, 4-6-1, Shirokanedai Minato-ku, Tokyo 108-8639, Japan.

E-mail: aito@ims.u-tokyo.ac.jp

${ }^{4}$ These authors contributed equally to this study.

Received 06 November 2007; revised 23 January 2008; accepted 28 January 2008
} 
Cell adhesion molecule 1 (CADM1) is the name currently approved by the HUGO gene nomenclature committee for the gene formerly referred to as spermatogenic immunoglobulin superfamily, ${ }^{4}$ tumor suppressor in lung cancer- $1,{ }^{5}$ synaptic cell adhesion molecule, ${ }^{6}$ nectin-like molecule- $2,{ }^{7}$ or immunoglobulin superfamily $4 \mathrm{~A}^{8}$ Structurally, this adhesion molecule has three immunoglobulin-like motifs in the extracellular domain and a short cytoplasmic domain that interacts with other proteins via the protein 4.1-binding and PDZbinding motifs. ${ }^{4,7}$ A variety of cells express CADM1, including epithelial cells, such as pulmonary and biliary epithelial cells, ${ }^{9,10}$ and non-epithelial cells, such as neurons, ${ }^{6}$ spermatogenic cells, ${ }^{4,11}$ and mast cells. ${ }^{12}$ CADM1 forms homodimers on the cell membrane through cis-interactions, and binds either trans-homophilically or heterophilically, depending on the cell type expressing the CADM1 and the binding partners available on adjacent cells. The trans-homophilic binding occurs among neurons ${ }^{6}$ and between mast cells and neurons, ${ }^{13}$ and the transheterophilic binding occurs between mast cells and fibroblasts $^{11}$ and between spermatogenic and Sertoli cells. ${ }^{8}$ In addition to the full-length form of approximately $100 \mathrm{kDa}$, CADM1 appears to have a shorter form of about $35 \mathrm{kDa}$, which is probably generated by $\mathrm{N}$-terminal truncation of the full-length form, as suggested in murine mast cells; ${ }^{12}$ however, the function of this truncated form remains unclear.

Bone marrow-derived cultured mast cells from wild-type mice express CADM1 abundantly. ${ }^{12}$ When these cells are injected into the peritoneal cavity of mast cell-deficient W/ $\mathrm{W}^{\mathrm{v}}$ mice, they not only survive in the cavity but also settle down into the mesentery. ${ }^{14}$ This settlement process is thought to include two events, adhesion of mast cells to mesothelial cells and transmigration of mast cells across mesothelial cells. We showed previously that mast cells use CADM1 to complete both events. ${ }^{15}$ CADM1 appears to play an important role in the interaction between mast cells and mesothelial cells. Besides mast cells, a variety of tumor cells seem to interact with mesothelial cells in vivo, because they often colonize on the serosal surface of the mesentery and pleura as primary or metastatic tumors. By analogy, CADM1 may be involved in tumor cell-mesothelial cell interactions when the tumor cells express CADM1.

In the present study, we first examined CADM1 expression in surgically resected MPMs and tumor-free pleurae via western blot and immunohistochemical analyses, and found that a considerable subset of MPMs expressed the full length, probably functional, form of CADM1, whereas non-neoplastic mesothelial cells did not express CADM1 at all. Second, we compared two MPM cell lines, one that expresses CADM1 and one that does not, by culturing them in soft agar and coculturing them on monolayers of either primary mesothelial cells or lung fibroblasts. Whereas CADM1 inhibited the anchorage-independent growth of MPM cells in soft agar, it promoted their adhesion and growth on mesothelial cells, a function that might explain why MPM cells prefer to grow on the pleural surface.

\section{MATERIALS AND METHODS}

\section{Human Samples}

The characteristics of 57 patients with MPM are shown in Table 1. All patients underwent surgical operations at the Hyogo Medical Center for Adults (Akashi, Japan; the former affiliation of M Okada), and none received any adjuvant

Table 1 Patient characteristics and CADM1 immunoreactivity

\begin{tabular}{ccc}
\hline Factor & $\begin{array}{c}\text { Total } \\
(n=57)\end{array}$ & CADM1 immunoreactivity \\
& $\begin{array}{c}\text { MS-positive } \\
(n=14)\end{array} \quad \begin{array}{c}\text { MS-negative } \\
(n=43)\end{array}$
\end{tabular}

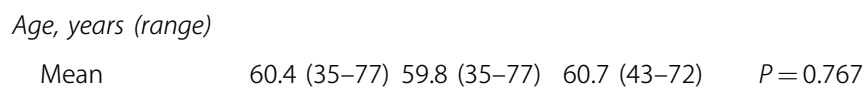

Sex, $n(\%)$

$\begin{array}{lrrrr}\text { Male } & 48(84.2) & 10(20.8) & 38(79.2) & P=0.136 \\ \text { Female } & 9(15.8) & 4(44.4) & 5(55.6) & \end{array}$

Histology, $n$ (\%)

$\begin{array}{lcccc}\text { Epithelioid } & 42(73.7) & 7(16.7) & 35(83.3) & \\ \text { Biphasic } & 11(19.3) & 3(27.3) & 8(72.7) & P=0.001 \\ \text { Sarcomatoid } & 4(7.0) & 4(100) & 0(0) & \end{array}$

Operation, $n(\%)$

$\begin{array}{lclrr}P / D^{a} & 31(54.4) & 8(25.8) & 23(74.2) & \\ E^{b} P^{b} & 21(36.8) & 6(28.6) & 15(71.4) & P=0.308 \\ I^{C} & 5(8.8) & 0(0) & 5(100) & \end{array}$

IMIG stage, $n(\%)^{d}$

$\begin{array}{lrlrl}\text { I } & 6(10.5) & 3(50.0) & 3(50.0) & \\ \text { II } & 12(21.1) & 3(25.0) & 9(75.0) & \\ \text { III } & 35(61.4) & 8(22.9) & 27(77.1) & \\ \text { IV } & 4(7.0) & 0(0) & 4(100) & \end{array}$

Cytology, $n(\%)^{\mathrm{e}}$

$\left.\begin{array}{lrrc}\text { Positive } & 24(42.1) & 5(20.8) & 19(79.2) \\ \text { Negative } & 20(35.1) & 7(35.0) & 13(65.0)\end{array}\right\} \quad P=0.293$

apleurectomy.

$\mathrm{b}_{\text {Extrapleural pneumonectomy. }}$

Incomplete resection.

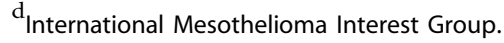

e Pleural effusions examined.

Not determined.

${ }^{\mathrm{g}}$ Not performed. 
therapies, such as irradiation and chemotherapy, before the operation. At least two pathologists with a specialty in pulmonary oncology reviewed paraffin-embedded blocks from formalin-fixed surgical specimens and definitively diagnosed each case based on currently accepted histopathologic criteria ${ }^{1}$ combined with immunohistochemical, clinical, and radiographic findings. In some cases, resected tumors were freshly cut into small cubes and frozen for protein analyses. The disease staging was according to the criteria of the International Mesothelioma Interest Group. Tumor-free lung parenchyma, pleura, and greater omenta were sampled from surgical materials of patients undergoing operations for diseases unrelated to MPM. Surgically resected materials were handled and analyzed in the present study according to the guidelines approved by the ethical committee of the Hyogo Medical Center for Adults.

\section{Cell Lines and Antibodies}

Human NCI-H28, NCI-H2052, and MeT-5A ${ }^{16}$ cells were purchased from the American Type Culture Collection (Manassas, VA, USA), Meso-1 and Meso-4 cells ${ }^{17}$ from Riken Cell Bank (Tsukuba, Japan), and TIG-1 cells ${ }^{18}$ from Health Science Research Resources Bank (Osaka, Japan). All cells were maintained in Dulbecco's modified Eagle's medium supplemented with $10 \%$ fetal calf serum. Cell doubling time was determined according to the method we described previously. ${ }^{19}$ Primary cultured mast cells were established from the bone marrow of C57/BL6 mice as described previously. ${ }^{12}$ To establish Meso-1 sublines that express CADM1 exogenously, the full-length cDNA for human CADM1 was obtained from human lung mRNA (Biochain, Hayward, CA, USA) by reverse transcription and polymerase chain reaction with a primer set of $5^{\prime}$-agtc tgaggcaggtgcccgacat- $3^{\prime}$ and $5^{\prime}$-cagttggacacctcattggaac- $3^{\prime}$. The double-stranded cDNA was subcloned into the Bluescript vector via the EcoRV site by TA cloning, and was determined by sequencing to have no mutations. The EcoRI-XhoI fragment containing the cDNA insert was subsequently subcloned into the pCX4bsr vector via the EcoRI and NotI site after the XhoI and NotI sites were blunt-ended with the Klenow large fragment. Meso-1 cells were transfected with either the resulting vector construct or the empty vector and were then selected by their resistance to blasticidin $S$ $(5 \mu \mathrm{g} / \mathrm{ml}$; Invitrogen, Carlsbad, CA, USA).

The rabbit polyclonal anti-CADM1 C-terminal antibody was generated previously. ${ }^{12}$ Other primary antibodies used in the present study were anti-D2-40 (DakoCytomation, Glostrup, Denmark), anti-calretinin (DakoCytomation), anti- $\beta$-actin (AC-74; Sigma Chemical, St Louis, MO, USA), and anti-vimentin (V-9; Thermo Fisher Scientific, Waltham, MA, USA). Peroxidase- and fluorescence-conjugated secondary antibodies were purchased from GE Healthcare (Little Chalfont Buckinghamshire, UK) and Jackson ImmunoResearch (West Grove, PA, USA), respectively.

\section{Immunohistochemistry and Immunocytochemistry}

The procedures for CADM1 immunodetection were described in detail previously. ${ }^{9}$ Briefly, formalin-fixed, paraffinembedded tissues were cut into sections (4- $\mu \mathrm{m}$ thick) air-dried overnight at $37^{\circ} \mathrm{C}$ deparaffinized in xylene, and rehydrated in a descending ethanol series. Endogenous peroxidase activity was blocked by immersion for $10 \mathrm{~min}$ in $0.3 \%$ hydrogen peroxide in methanol followed by a single wash in phosphate-buffered saline (PBS), $\mathrm{pH}$ 7.4. After the sections were autoclaved for $15 \mathrm{~min}$ at $121^{\circ} \mathrm{C}$ in $10 \mathrm{mM}$ citrate buffer solution ( $\mathrm{pH}$ 6.0), they were incubated with the anti-CADM1 antibody (1:200 dilution), followed by incubation with a biotin-conjugated second antibody. The color was developed using 3,3-diaminobenzidine (DAB; Sigma Chemical) as the chromogen. The slides were then counterstained with Mayer hematoxylin, dehydrated, and mounted. Negative controls were prepared by substituting nonimmune rabbit serum or control mouse IgG for the specific primary antibody. The procedures for immunodetection of D2-40 and calretinin were similar to the above, but included some modifications, as described previously. ${ }^{20}$

For immunocytochemistry, cells were cultured in chamber slides (Nalge Nunc International, Naperville, IL, USA) and processed as described previously. ${ }^{13}$ Briefly, methanol-fixed cultured cells were singly immunostained with the antiCADM1 antibody followed by a Cy2-conjugated anti-rabbit IgG antibody, or doubly immunostained with a combination of anti-CADM1 and anti-calretinin antibodies, followed by $\mathrm{Cy} 2$-conjugated anti-rabbit and $\mathrm{Cy} 3$-conjugated anti-mouse IgG secondary antibodies, respectively.

\section{Western Blot Analysis}

Frozen samples of lung parenchyma, parietal pleura, and MPMs were crushed into pieces and vigorously vortexed in a buffer solution containing $50 \mathrm{mM}$ Tris- $\mathrm{HCl}(\mathrm{pH}$ 8.0), $150 \mathrm{mM} \mathrm{NaCl}, 1 \%$ Triton X-100, and a protease inhibitor cocktail (Sigma Chemical). Cultured cells were lysed in the same buffer by scraping, pipetting, and vortexing. Impurities were removed by centrifugation at $12000 \mathrm{~g}$. The resulting lysates were separated on 10\% SDS-polyacrylamide gels and transferred to Immobilon membranes (Millipore, Bedford, MA). The blots were reacted with the indicated primary antibodies in a buffer containing $20 \mathrm{mM}$ Tris- $\mathrm{HCl}$ ( $\mathrm{pH} 8.0$ ), $150 \mathrm{mM} \mathrm{NaCl}, 0.05 \%$ Tween 20, and 5\% skim milk (Difco, Sparks, MD, USA), and then with appropriate peroxidaseconjugated secondary antibodies in the same buffer. The luminescence was developed with ImmunoStar reagents (Wako, Osaka, Japan) before exposure. After the blots were stripped, they were probed again with an anti- $\beta$-actin antibody according to similar procedures.

\section{Colony Formation Assay}

To measure colony formation, $5 \times 10^{4}$ cells were suspended in $0.3 \%$ soft agar and immediately plated onto $35-\mathrm{mm}$ dishes precast with $0.72 \%$ agar. Cells were incubated at $37^{\circ} \mathrm{C}$ and fed 
weekly by overlaying the soft agar with fresh medium. Two weeks after plating, 50 independent rectangular areas $\left(3 \mathrm{~mm}^{2}\right.$ each) of the culture were photographed, and the total numbers of colonies with a diameter larger than $100 \mu \mathrm{m}$ were counted. The mean and standard deviation of colony numbers per area were calculated from triplicate cultures for each experimental group.

\section{Coculture of MPM Cells with Primary Mesothelial Cells or TIG-1 Cells}

Following the method that we described previously to isolate mouse mesenteric mesothelial cells, ${ }^{15}$ human mesothelial cells were freshly isolated from the surgically resected greater omenta. Briefly, transparent sheet-like areas of the omenta were excised with scalpels and were then incubated in $0.25 \%$ trypsin-EDTA solution (Sigma) at $37^{\circ} \mathrm{C}$ for $30 \mathrm{~min}$. After fatty and fibrous tissue masses were removed by filtration through a mesh sieve, cells suspended in the solution were collected by centrifugation, and were cultured in Dulbecco's modified Eagle's medium supplemented with $10 \%$ fetal calf serum. Approximately $2 \times 10^{4}$ viable cells were seeded into each well of 12-well plates; the cells grew to confluence in 4 days. TIG-1 cell monolayers were prepared with similar procedures.

To label living cells with fluorescence, MPM cells at semiconfluency were incubated for $30 \mathrm{~min}$ in medium containing the tracer DiI (Molecular Probe, Eugene, OR, USA) at a concentration of $10 \mu \mathrm{M}$. Cells were harvested by trypsinization and washed with PBS 3 times. After we confirmed that nearly all of the harvested cells were evenly labeled with DiI, we seeded the labeled cells onto mesothelial or TIG-1 cell monolayers covering the bottom of 12 -well plates. We seeded $5 \times 10^{3}$ and $1 \times 10^{3}$ DiI-labeled cells in the experiments assessing adherent cell morphology and growth kinetics, respectively. The cocultures were observed every day under a fluorescence microscope (CKX4; Olympus, Tokyo, Japan) equipped with a CCD camera (DP70; Olympus). To assess the growth kinetics, the total number of DiI-containing cells detected in 30 randomly selected high-power fields ( 1 field is approximately $0.13 \mathrm{~mm}^{2}$ ) was counted, and the mean and s.d. per field was calculated from triplicate cocultures for each experimental group. Experiments were repeated 3 times with similar results. In some cases, unlabeled MPM cells were seeded onto mesothelial cell monolayers and subjected to immunocytochemistry.

\section{Statistics}

The $t$-test was used to analyze colony formation and growth kinetics and the $\chi^{2}$ test was used for the analyses of clinicopathologic and immunohistochemical correlation. The statistical tests were performed using StatView software (Abacus Concepts Inc., Cary, NC, USA) on a Macintosh computer. A $P$-value $<0.05$ was considered to be significant.

\section{RESULTS \\ Expression of the Full-Length Form CADM1 in MPMs, but not in Non-Neoplastic Mesothelial Cells}

We examined CADM1 expression in tumor-free pleurae and MPMs via immunohistochemistry using an antibody against the CADM1 C terminus. Non-neoplastic mesothelial cells, whether morphologically normal cells or those with reactive atypia, were stained weakly and diffusely in the cytoplasm (Figure 1a and b). In contrast, there was strong membranous staining (MS) in the terminal bronchiolar epithelium, where CADM1 signals were localized primarily on the lateral cell membrane (Figure 1a). Fifty-seven surgically resected MPMs were immunostained according to the same procedures (Table 1). The majority of MPMs showed cytoplasmic staining similar to that of non-neoplastic mesothelial cells (Figure 1c), but in some tumors, CADM1 signals were detected predominantly on the cell membrane (Figure $1 \mathrm{~d}$ and e). We divided the 57 MPMs into 2 groups according to the staining pattern: (1) MS-positive MPMs $(n=14)$, in which the MS signals were dominant and as clear as in respiratory epithelial cells, and (2) MS-negative MPMs $(n=43)$, which included all other samples. The MS-negative group consisted of 37 tumors with enhanced cytoplasmic signals and six tumors with cytoplasmic signals that were comparable to or weaker than those of non-neoplastic mesothelial cells. In MS-positive MPMs, MS signals were detected not only on the tumor cells but also between tumor cells and non-neoplastic mesothelial cells (Figure 1f and g).

We examined whether this grouping of MPMs by CADM1 immunohistochemistry correlated with any clinicopathologic parameters, including immunoreactivity for D2-40 and calretinin, the most specific diagnostic marker set for MPMs. The CADM1 MS-positive group was closely correlated with the sarcomatoid histologic subtype (Table 1). In contrast, three of the four sarcomatoid tumors were negative for D2-40 and calretinin (Table 2). Thus, the CADM1MS-positive group was correlated with negative immunoreactivity for D2-40 and calretinin (Table 2). Although there was no significant correlation between CADM1 expression and any other clinicopathologic parameters available (Table 1), the number of MS-negative MPMs judged cytologically as negative was just one-third (13/38), whereas more than half of MSpositive MPMs were judged to be cytologically negative (7/13; Table 1).

To further characterize the subset of MS-positive MPMs, we used western blot analyses to examine the CADM1 expression in four of the tumors, 4MS-negative MPMs, tumorfree parietal pleurae, and the lung parenchyma, which is known to express high levels of CADM1. ${ }^{9}$ In MPMs and the lung parenchyma, two immunoreactive bands were detected at mobility sizes of 100 and $35 \mathrm{kDa}$ (Figure $1 \mathrm{~h}$ ). We considered the larger band to represent the full-length form of CADM1 and the smaller its truncated form, as we described in mast cells. ${ }^{12}$ Four MS-positive MPMs expressed the fulllength form as abundantly as the lung parenchyma, whereas 


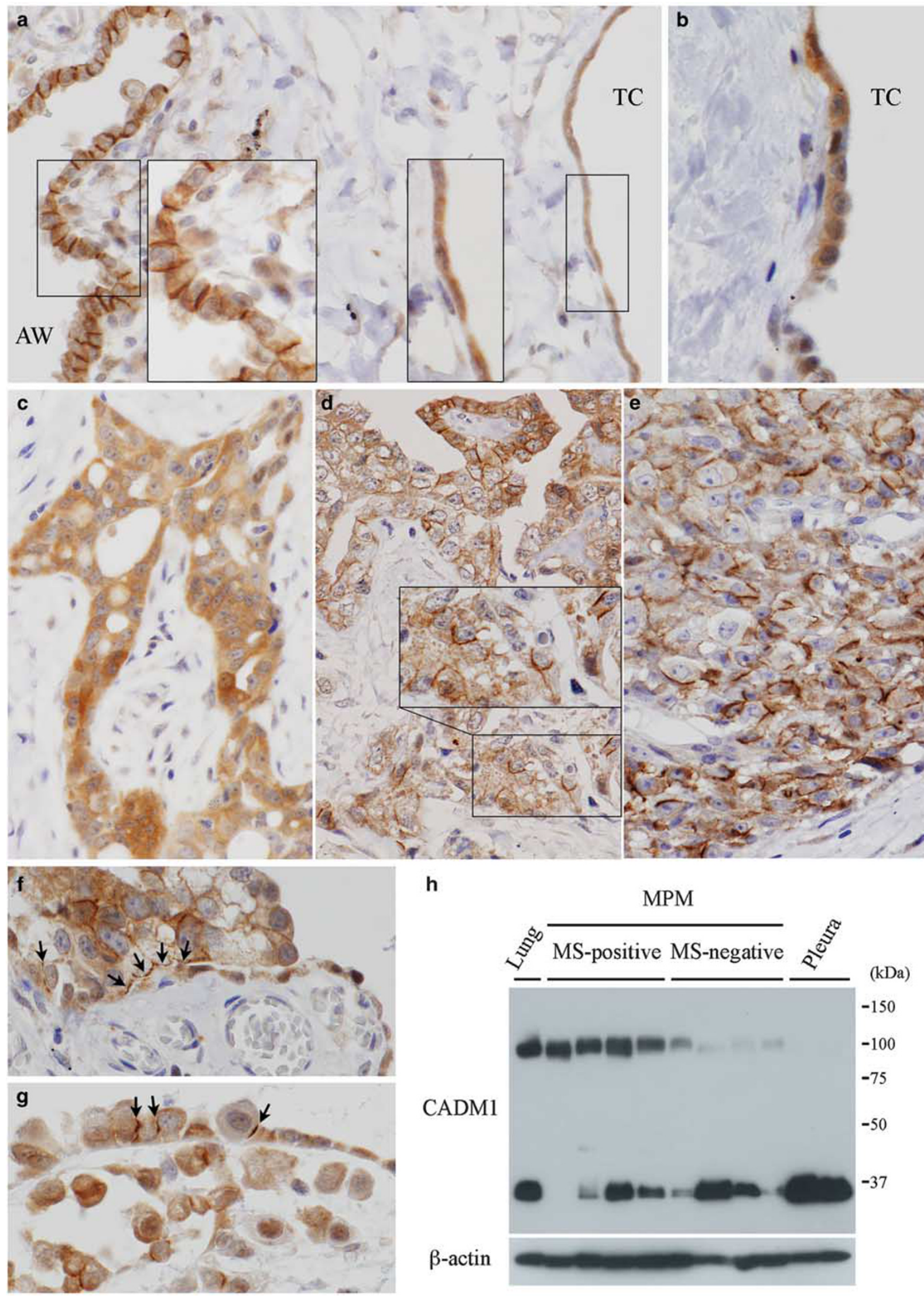


4MS-negative MPMs expressed it rather weakly (Figure 1h). The levels of the truncated form varied among tumors, but tended to be higher in MS-negative MPMs. Tumor-free parietal pleurae expressed the truncated form strongly, but not the full-length form at all (Figure 1h). Three MS-positive MPMs were subjected to direct sequencing and were found to have no mutation in the coding region of the CADM1 mRNA.

\section{CADM1 Inhibits Anchorage-Independent Growth of MPM Cells}

We used western blot analyses to examine CADM1 expression in four MPM-derived cell lines (NCI-H28, NCI-H2052, Meso-1, and Meso-4) and one non-neoplastic mesothelial cell-derived line transformed by SV40 T antigen (MeT-5A). ${ }^{16}$ NCI-H28 cells expressed the full-length form of CADM1 at high levels nearly comparable to those in lung parenchyma and cultured mouse mast cells, whereas NCI-H2052 and Meso-4 cells expressed it at lower but still appreciable levels (Figure 2A). In contrast, neither Meso-1 nor MeT-5A cells expressed CADM1 at all (Figure 2A). The variable mobility of CADM1 proteins detected among different cell lines can probably be attributed to post-transcriptional regulation, such as differential levels of glycosylation, as we previously reported in the lung ${ }^{9}$ and liver. ${ }^{10}$ Subcellular localization of CADM1 was examined by immunocytochemistry. CADM1 signals were clearly detected on the peripheral margin of NCI-H28 cells (Figure 2Ba), through which the cells were in membrane-membrane contact with each other, whereas faint signals were detectable only in the cytoplasm, and not on the cell membrane, of Meso-1 cells (Figure 2Bb).

We next subjected these cells to colony formation assays in soft agar. Meso-1 and MeT-5A cells formed the largest and second largest number of colonies, respectively, whereas NCI$\mathrm{H} 28$ cells were incapable of forming colonies (Figure 2Ca, b and d). NCI-H2052 and Meso-4 cells did form colonies but in significantly smaller numbers than Meso-1 cells (Figure $2 \mathrm{Cd}$ ). Because the ability of the cell lines to form colonies seemed to be inversely correlated with their levels of CADM1 expression, we examined whether CADM1 inhibits anchorage-independent growth of MPMs. We obtained 2 Meso-1 subclones, Meso-1-CADM1-Hi and Lo, which express the full-length form CADM1 exogenously at high and low levels, respectively (Figure 2A). Meso-1-CADM1-Hi cells expressed CADM1 on the membrane surface (Figure $2 \mathrm{Bc}$ ) and had little ability to form colonies, whereas Meso-1-CADM1-Lo cells formed a countable but markedly smaller number of colonies than the parental cells (Figure 2Cc and d). In

Table 2 Immunohistochemical phenotype of MPMs: correlation between CADM1 and D2-40 or calretinin

\begin{tabular}{|c|c|c|c|c|c|c|c|}
\hline & \multirow[t]{2}{*}{ No. of Cases } & \multicolumn{2}{|c|}{ D2-40 } & \multirow[t]{2}{*}{$P$-value } & \multicolumn{2}{|c|}{ Calretinin } & \multirow[t]{2}{*}{$P$-value } \\
\hline & & Positive & Negative & & Positive & Negative & \\
\hline MPM & 57 & 48 & 9 & & 48 & 9 & \\
\hline \multicolumn{8}{|c|}{ Histologic subtype } \\
\hline Sarcomatoid & 4 & 1 & 3 & & 1 & 3 & \\
\hline \multicolumn{8}{|l|}{ CADM1 $\mathrm{MS}^{\mathrm{a}}$} \\
\hline Positive & 14 & 8 & 6 & 0.001 & 9 & 5 & 0.019 \\
\hline Negative & 43 & 40 & 3 & & 39 & 4 & \\
\hline
\end{tabular}

\footnotetext{
${ }^{\mathrm{a}}$ Membranous staining.
}

Figure 1 Expression of CADM1 in MPMs. (a-g) Immunohistochemical staining of CADM1 in human lungs and MPMs. Paraffin sections were prepared from nearly normal visceral pleura (a), parietal pleura covered by a single layer of mesothelial cells with reactive atypia (b), and MPMs of the epithelioid (c, $\mathbf{f}$, and $\mathbf{g}$ ) and sarcomatoid (d and $\mathbf{e}$ ) subtypes. Sections were reacted with an anti-CADM1 antibody, and the immunostaining was visualized with DAB. The nuclei were counterstained with hematoxylin. Boxed regions were enlarged to show the subcellular localization of CADM1 in bronchiolar cells (a, left), pleural mesothelial cells (a, right), and epithelioid MPM cells (d). Arrows in $\mathbf{f}$ and $\mathbf{g}$ indicate CADM1 signals localized on MPM cell membranes facing non-neoplastic mesothelial cells. AW, airway; TC, thoracic cavity. Original magnification: $\times 400$, except for b, $\times 500$. (h) Western blot analysis of CADM1 in MPMs and pleura. Lysates of lung parenchyma (leftmost lane), MPMs (four each for MS-positive and -negative tumors), and parietal pleura (two rightmost lanes) were electrophoresed in a 10\% SDS-polyacrylamide gel and blotted with the anti-CADM1 antibody. After the blot was stripped, it was probed again with an anti- $\beta$-actin antibody to indicate the relative amount of protein in each lane. 
addition, ectopic CADM1 lengthened the cell-doubling time of Meso-1 cells (Figure 2Cd), as is the case with esophageal and nasopharyngeal cancer cell lines. ${ }^{21,22}$ Vector-control subclones (Meso-1-Vec-1 and -2) did not differ from the original Meso-1 cells in colony formation and cell-doubling time (Figure 2Cd).

A

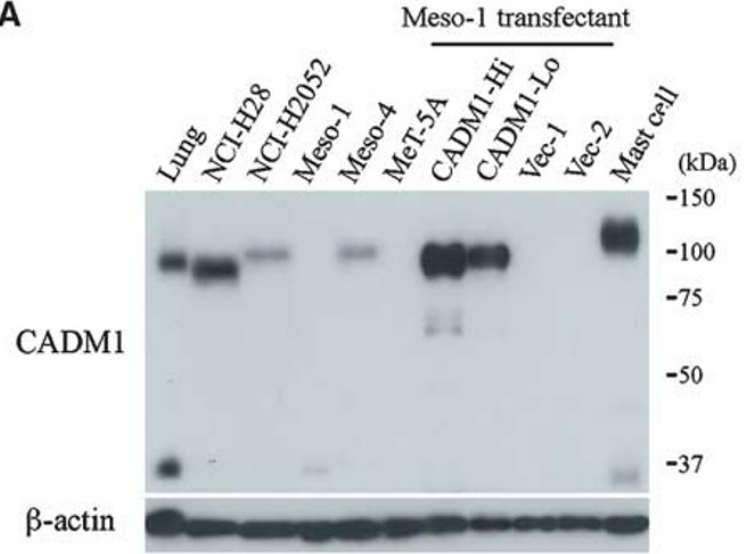

B
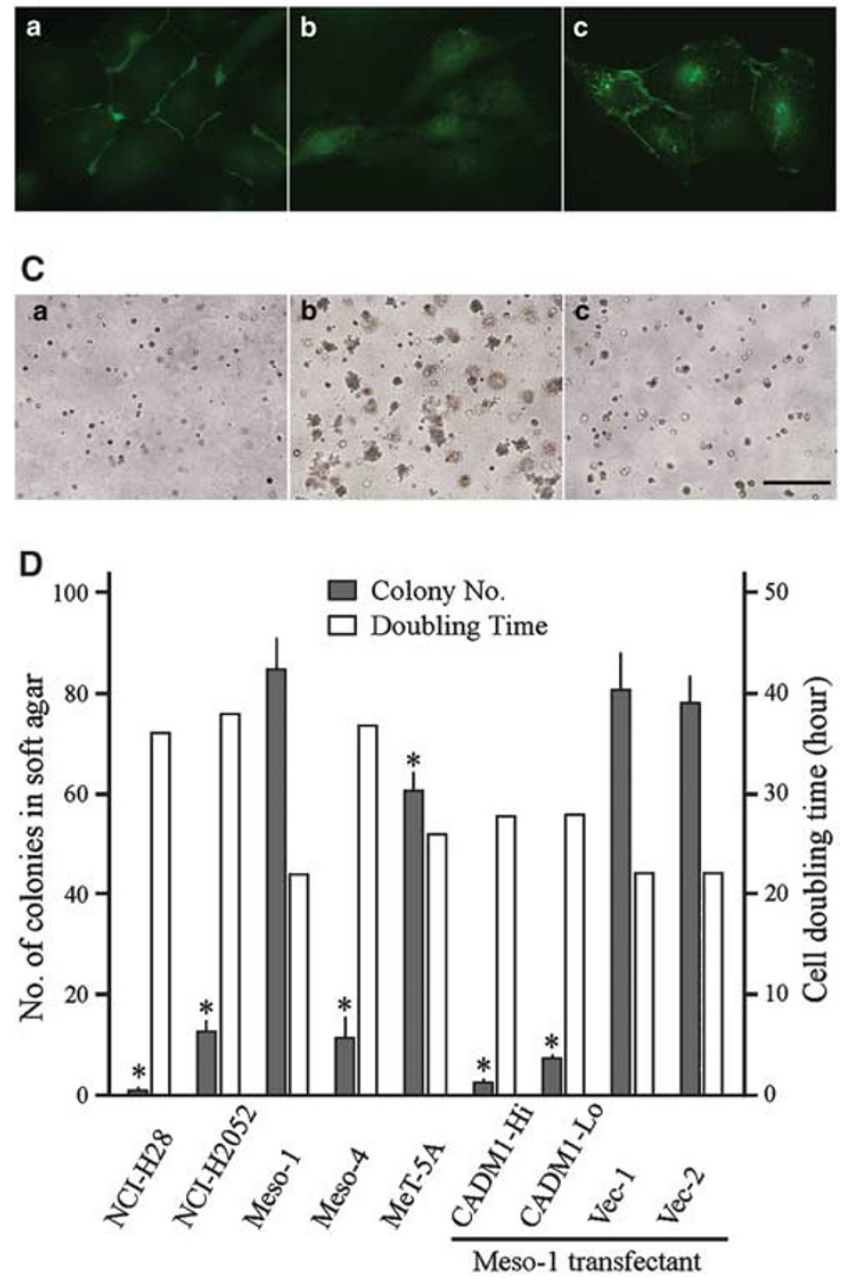

Involvement of CADM1 in Adhesion between Cocultured MPM and Primary Mesothelial Cells

The gross features of MPM suggest that interactions between MPM cells and neighboring mesothelial cells occur inevitably and repeatedly during tumor growth and that these interactions are a key event for MPM dissemination over the pleural surface. As shown in Figure if and g, we actually detected CADM1 signals on the cell membrane of MPM cells adjacent to non-neoplastic mesothelial cells, even in advanced tumors. To examine what roles CADM1 plays in the interaction between MPM and mesothelial cells, we compared CADM1-positive and -negative MPM cells in coculture with primary mesothelial cells. We freshly isolated mesothelial cells from surgically resected greater omenta and seeded the cells onto culture dishes to yield cell monolayers in a few days. We considered the monolayers to consist of mesothelial cells because the cultured cells were polygonal and flattened in shape and were immunologically positive for the mesothelial markers calretinin and $\mathrm{D} 2-40,{ }^{20}$ but negative for vimentin, a fibroblastic marker (Figure $3 \mathrm{~A}$ and $\mathrm{B}$ ). ${ }^{23}$ These cells maintained their confluency for about a week, probably because of contact inhibition, and lacked full-length CADM1 (Figure 3B), consistent with the western blot result on the pleura (see Figure $1 \mathrm{~h}$ ).

We labeled NCI-H28 and Meso- 1 cells with the fluorescent tracer DiI and seeded them onto the mesothelial cell monolayers. The numbers of DiI-labeled cells adherent to the monolayers were comparable between the two cocultures the next day, but the two types of MPM cells clearly exhibited distinct cell morphologies. Representative photomicrographs of the 2-day-long cocultures are shown in Figure $3 \mathrm{Ca}$ and $\mathrm{b}$. Individual NCI-H28 cells had a well-spread and flattened cell shape, whereas Meso-1 cells were round. Such morphologic differences remained detectable as the MPM cells proliferated on the monolayers in the following several days. NCI-H28 cells became scattered on the monolayer and sometimes

Figure 2 Expression of CADM1 in MPM cell lines and its inhibitory effect on colony formation. (A) Western blot analysis of CADM1 in various types of MPM cells. Lysates of the indicated cells were electrophoresed in a $10 \%$ SDS-polyacrylamide gel and blotted with the anti-CADM1 antibody. As positive controls, lysates of lung parenchyma and cultured mouse mast cells were loaded in the leftmost and rightmost lanes, respectively. The blot was stripped and probed again with the anti- $\beta$-actin antibody as a loading control. (B) Immunodetection of CADM1 in NCl-H28 (a), Meso-1 (b), and Meso-1-CADM1-Hi (c) cells. Cells were fixed with methanol and were incubated serially with the anti-CADM1 primary antibody and Cy2-conjugated secondary antibody. (C) Anchorage-independent growth and cell doubling times of various MPM cells. Cells were left to grow in soft agar for 2 weeks, and were photographed through a $4 \times$ objective. Representative photomicrographs are shown: (a), NCl-H28; (b), Meso-1; (c), Meso-1-CADM1-Hi. Bar $=0.5 \mathrm{~mm}$. The average numbers of colonies $(>100 \mu \mathrm{m})$ per field were calculated from triplicate cultures, and are plotted as gray columns in (D), with short bars indicating standard deviations. Cell doubling time was measured by plating cells onto standard culture dishes and is plotted as white columns, also in (D). ${ }^{\star} P<0.05$ by $t$-test when compared with the value of Meso-1 cells. 
formed a cell nest, whereas Meso-1 cells piled up on the monolayer (Figure 3Cd and e). When we labeled Meso-1CADM1-Hi cells with DiI and seeded them onto mesothelial cell monolayers, they resembled NCI-H28 cells rather than Meso-1 cells: they had a well-spread cell shape and grew scattered across the monolayer, instead of piling up (Figure 3Cc and f). We double-stained the cocultures of unlabeled MPM cells on mesothelial cell monolayers with antibodies against CADM1 and calretinin. Consistent with the results of the western blot analyses, primary mesothelial cells were positive for calretinin, but negative for CADM1 (Figure 3B and $\mathrm{Cg}-\mathrm{i}$ ). In NCI-H28 and Meso-1-CADM1-Hi cells, CADM1 signals were concentrated on the plasma membrane at the cell margin, whereas Meso- 1 cells had faint signals only in the cytoplasm (Figure $3 \mathrm{Cg}-\mathrm{i}$ ). Vector-control Meso-1 sublines behaved similarly to the parental cells in this coculture model (data not shown).

We prepared another coculture model in which MPM cells were seeded onto monolayers composed of TIG-1 cells, which are non-immortalized diploid fibroblasts derived from human lung and which are known to maintain their confluency for several days because of contact inhibition. ${ }^{18,24}$ TIG-1 cells expressed high levels of vimentin, a fibroblastic marker, but their CADM1 level was negligible in comparison with cultured mouse mast cells (Figure 3B), as is the case with NIH/3T3 fibroblasts. ${ }^{12}$ NCI-H28, Meso-1, and Meso-1CADM1-Hi cells were labeled with DiI, and then seeded onto TIG-1 cell monolayers. In the three cocultures, the number of adherent DiI-labeled cells was comparable, and there was no remarkable morphologic difference among the three types of MPM cells (Figure 3Cj-k), indicating that CADM1 was not involved in adhesion of MPM cells to TIG-1 fibroblasts.

\section{CADM1 Promotes Growth of MPM Cells on Primary Mesothelial Cell Monolayers}

We cocultured MPM cells on primary mesothelial cell monolayers, and examined the growth rates of DiI-labeled NCI-H28 and Meso-1 cells by counting the number of DiI-containing cells adherent to mesothelial cells every day. The monitoring period was limited to 4 days, because after 5 days or more of coculture, DiI fluorescence intensity within individual cells became too low to recognize all the cells derived from originally DiI-labeled cells. The increase in cell number was comparable between NCI-H28 and Meso-1 cells the day after the initiation of coculture, but in the following 3 days, the number of NCI-H28 cells was significantly larger than that of Meso-1 cells (Figure 4, upper). Meso-1-CADM1Hi cells grew still faster than NCI-H28 cells, whereas the growth rate of Meso-1-Vec-1 cells was similar to that of Meso-1 cells (Figure 4, upper). Contrasting results were obtained when these MPM cells were cocultured on monolayers of TIG-1 cells. The four types of MPM cells grew much more slowly than on mesothelial cell monolayers (Figure 4, lower). In addition, the growth rates of these cells were similar to each other, except that NCI-H28 cells grew more slowly after 3 days of coculture, probably reflecting their longer cell-doubling time (Figures 2Cd and 4, lower).

\section{DISCUSSION}

In the present study, we examined CADM1 expression in 57 surgically resected MPMs, and found that a quarter of the tumors expressed CADM1, probably in its full-length functional form, on the cell membrane. Colony formation assays suggested that the full-length form of CADM1 was a potential tumor suppressor of MPMs, because its expression levels were inversely correlated with the anchorage-independent growth of MPM cell lines. Similar characterizations of CADM1 have been reported in a variety of tumors, including non-small cell lung cancer ${ }^{25}$ and nasopharyngeal carcinoma. ${ }^{22}$ Because the cells of origin of these tumors, pulmonary and nasopharyngeal epithelial cells, ${ }^{9,22}$ express the full-length form of CADM1, it has been postulated that the loss of CADM1 initiates the transformation of these cells into neoplastic cells. In contrast, MS-positive MPMs seemed to acquire CADM1 expression during or after the process of malignant transformation from mesothelial cells, because non-neoplastic mesothelial cells, even those with reactive atypia, did not express the full-length form of CADM1. This expression profile is reminiscent of that of adult T-cell leukemia cells, which express CADM1 at much higher levels than non-neoplastic T cells. ${ }^{26}$ In this type of tumor, CADM1 is assumed to contribute to highly invasive phenotypes by mediating adhesion between leukemia and endothelial cells. Further study is needed to clarify whether CADM1 actually plays a role as a tumor suppressor during the development of MPMs in vivo.

Although mesothelial cells did not express the full-length form of CADM1, their cytoplasm was immunoreactive to the anti-CADM1 C-terminal antibody in the cytoplasm (Figure 1a and $\mathrm{b}$ ). In addition, the antibody detected a 35-kDa molecule in a lysate of tumor-free pleurae (Figure $1 \mathrm{~h}$ ), suggesting that the $35-\mathrm{kDa}$ molecule might be the source of the cytoplasmic stain in mesothelial cells. As we reported previously in murine mast cells, ${ }^{12}$ this molecule seems to be produced from an mRNA identical to that used to produce the full-length CADM1. Its identity is now under intensive investigation in our laboratory. According to our unpublished data (M Hagiyama and A Ito), the $35-\mathrm{kDa}$ molecule seems to be a C-terminal fragment of the full-length CADM1 produced by ectodomain shedding, and thus drastic changes in this shedding mechanism may be responsible for the expression of the full-length CADM1 in MPMs. MS-positivity was significantly correlated with the sarcomatoid subtype and negativity for D2-40 and calretinin. Sarcomatoid-subtype MPMs are often negative for D2-40 and calretinin ${ }^{20}$ and are therefore devoid of highly sensitive and specific diagnostic markers. Although the precise molecular mechanism of CADM1 expression in MPMs remains to be clarified, CADM1 immunohistochemistry may be useful for avoiding misdiagnosis of MPMs. 
A

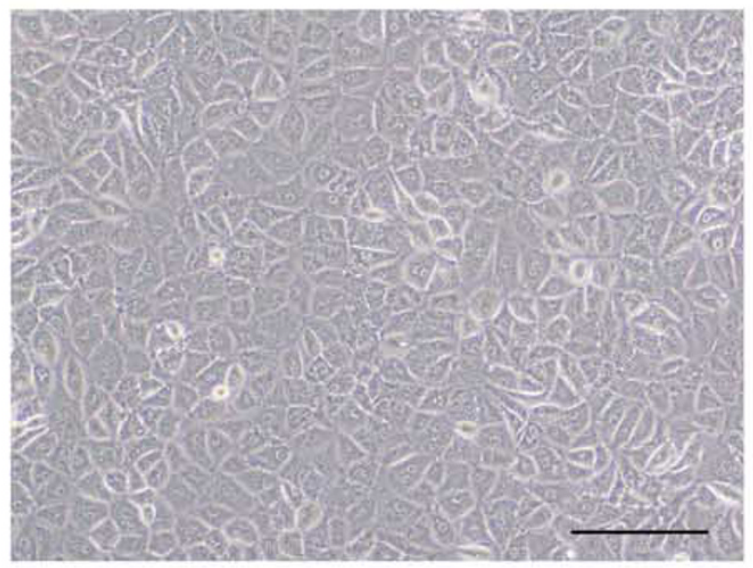

B

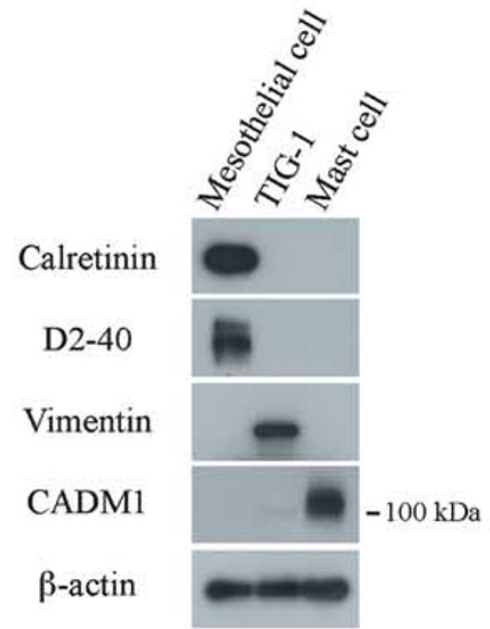

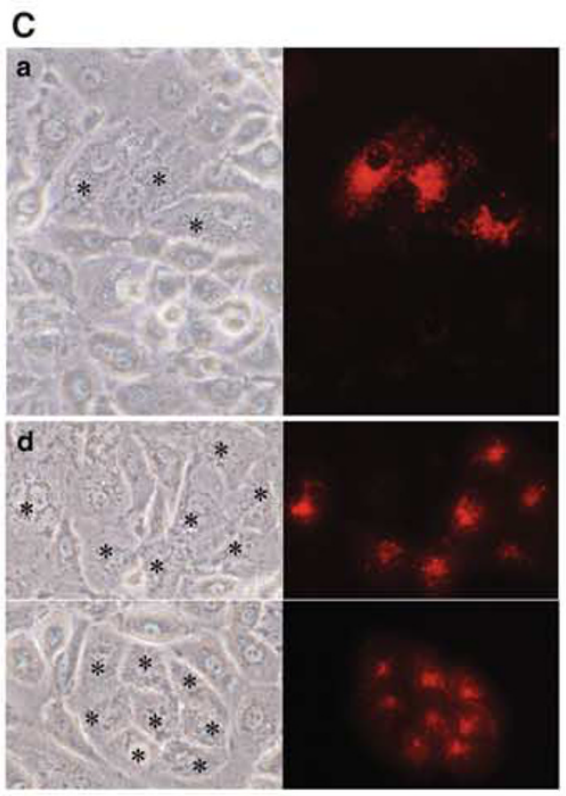
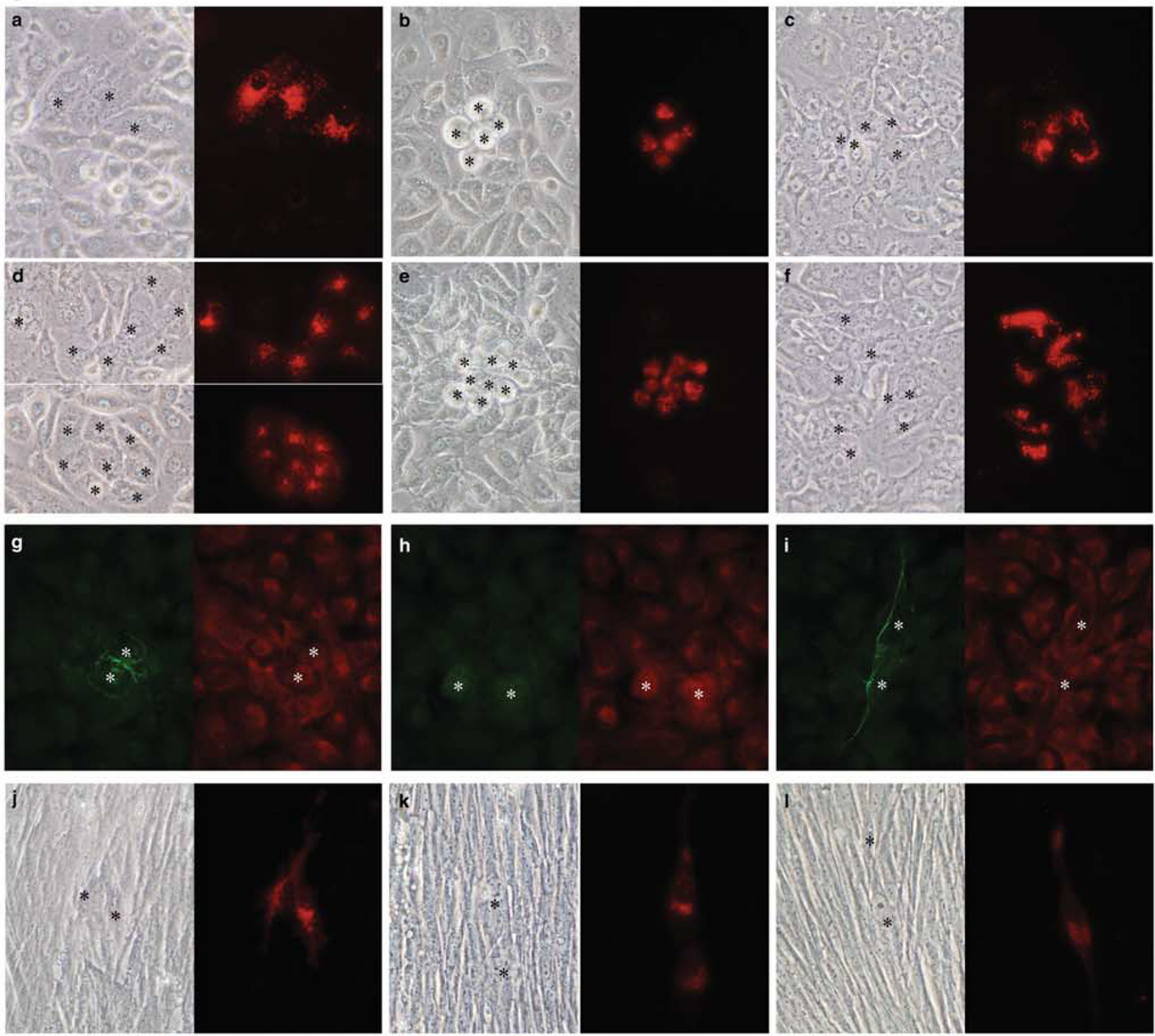
Full-length CADM1 appeared to play contrastive roles when MPM cells were cultured within soft agar and on primary mesothelial cell monolayers. Within soft agar, CADM1 inhibited anchorage-independent growth of MPM cells, whereas it promoted the growth of MPM cells on mesothelial cells. We previously obtained similar findings when we cultured mast cells in suspension and on fibroblastic cell monolayers. Ectopic CADM1 suppressed mast cell growth in suspension culture, whereas it supported mast cell growth and survival on fibroblastic cell monolayers. ${ }^{12,27}$ Colony formation assays of MPM cells and suspension culture of mast cells are similar in that CADM1 is thought to mediate homotypic cell aggregation by trans-homophilic binding. In contrast, in the two coculture models of MPM and mast cells, CADM1 is thought to mediate heterotypic cell-cell adhesion by trans-heterophilic binding. Therefore, CADM1 seems to generate distinct intracellular signals depending on whether it binds through trans-homophilic or heterophilic interactions.

MPM is classically portrayed as a diffuse tumor involving all pleural surfaces and encasing the lung and chest wall, and as the tumor grows it spreads to involve the parietal and visceral pleura confluently with little severe infiltration of lung parenchyma. ${ }^{1}$ Consistent with these gross features, MPM cells grew on primary mesothelial cells at much higher rates than on TIG-1 lung fibroblasts. In addition, we found a clear phenotypic difference between CADM1-positive and -negative MPM cells when we cultured these cells on mesothelial cell monolayers, but not on TIG-1 fibroblasts. CADM1-positive MPM cells spread more widely on mesothelial cells, grew in a scattered pattern over the mesothelial cells, and proliferated at higher rates. The full-length CADM1 appeared to promote efficient adhesion and growth of MPM cells specifically when it mediated the interaction of MPM cells with mesothelial cells. This function of CADM1 is likely to help MPM cells interact with neighboring non-neoplastic mesothelial cells and subsequently scatter over the pleural surface. The fact that a higher proportion of MS-positive MPMs from our patients were cytologically negative for pleural effusion may reflect the less frequent detachment of MS-positive cells from the pleural surface. Nonetheless, MS-positive MPMs were just a minor subset of our 57 samples, and we could not identify morphologic differences between MS-positive and -negative MPMs in the gross features we examined here. This may be largely because the tumors we examined in the present study were already rather highly advanced at the time of admission, and the occurrence of MPM-mesothelial cell interactions is likely to be limited to
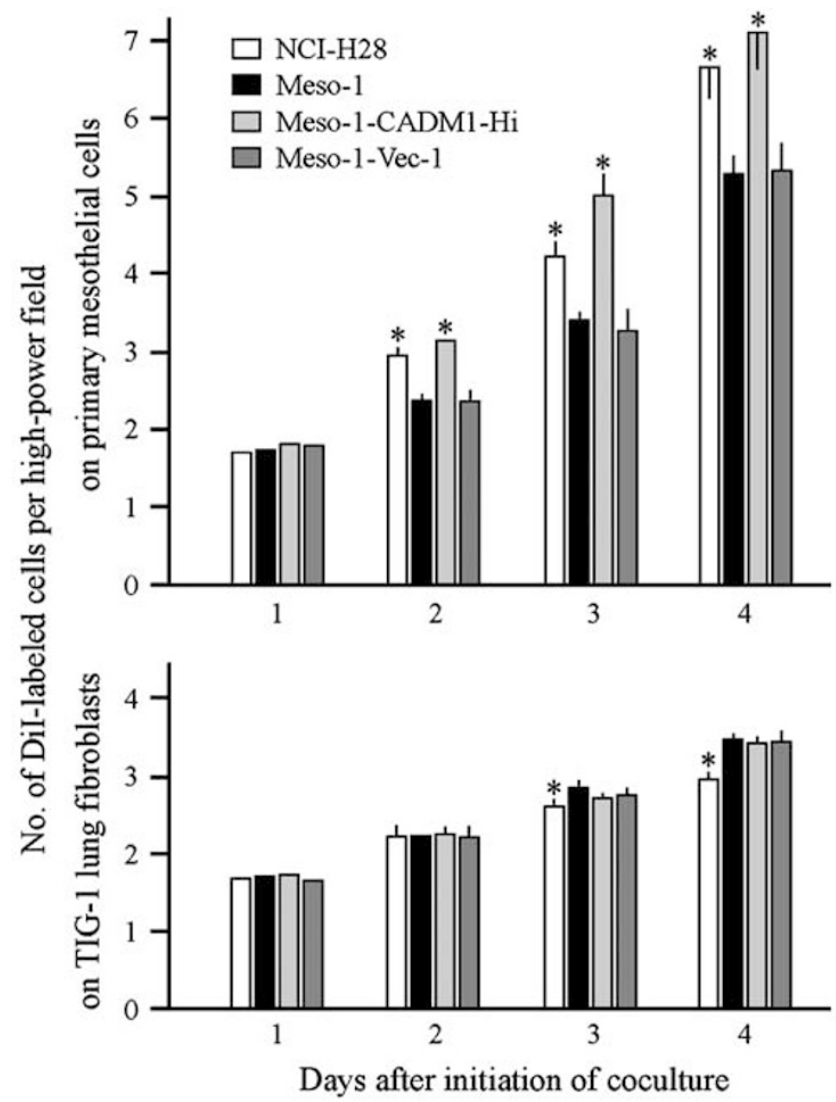

Figure 4 Growth kinetics of MPM cells on monolayers of primary mesothelial cells or TIG-1 lung fibroblasts. The four types of MPM cells indicated were labeled with Dil and seeded onto monolayers composed of either primary mesothelial cells (upper graph) or TIG-1 lung fibroblasts (lower graph). Every day after the initiation of the coculture, the total number of Dil-containing cells contained in 30 high-power fields were counted under a fluorescence microscope. The average numbers per field were calculated from triplicate cocultures and are plotted in columns, with short bars indicating standard deviations. For some points, the standard deviations were too small to be shown by bars. ${ }^{\star} P<0.05$ by $t$-test when compared with the value of Meso-1 cells on the identical day.

Figure 3 Coculture of MPM cells on primary mesothelial cell monolayers. (A) Phase-contrast photomicrograph of a primary mesothelial cell monolayer. Freshly isolated mesothelial cells from the greater omenta were seeded into 12-well plates and grew confluent in 4 days. The cells maintained their confluency for about a week. Bar $=100 \mu \mathrm{m}$. (B) Western blot analysis of cell type-specific markers in primary mesothelial cells and TIG-1 fibroblasts. Lysates of primary mesothelial cells, TIG-1 cells, and cultured mouse mast cells (positive control for CADM1) were electrophoresed in a 10\% SDS-polyacrylamide gel and blotted with the antibodies indicated. The blot was stripped and probed again with the anti- $\beta$-actin antibody as a loading control. (C) Morphologic comparison of CADM1-positive and -negative MPM cells growing on monolayers composed of either primary mesothelial cells (a-i) or TIG-1 lung fibroblasts (j-I). MPM cells were labeled with Dil (a-f, and j-l), and were seeded onto the cell monolayers. After 2 (a-c, and j-l) and $3(d-f)$ days, Dil-containing cells were detected under a fluorescence microscope. In each panel, the left and right are a phase-contrast and a Dil fluorescence image, respectively, of the identical field. In $d$, two distinct patterns of cell growth are shown. For immunofluorescence ( $\mathrm{g}-\mathrm{i})$, unlabeled MPM cells were seeded onto mesothelial cell monolayers. After 2 days, the cocultures were double-stained with anti-CADM1 and calretinin antibodies, and were visualized with Cy2 (left) and Cy3 (right), respectively. NCI-H28 cell cocultures are shown in a, d, g, and j; Meso-1 cell cocultures in b, e, h, and k; and Meso-1-CADM1-Hi cell cocultures in C, f, i, and I. Black and white asterisks indicate MPM cells labeled with Dil and anti-CADM1 antibody, respectively. 
relatively early stages of MPM development. Alternatively, MS-negative MPMs may actually contain a few MS-positive tumor cells, as these tumors expressed weak but detectable levels of the full-length CADM1 when assessed with western blot analyses (Figure 1h). Although the molecular and cellular changes that precede the appearance of macroscopically overt MS-positive and -negative MPMs remains to be discovered, the present study is, to our knowledge, the first report that identifies a molecular basis for the gross features characteristic of MPM, and suggests that CADM1 promotes the growth of MPM cells on the pleural surface by specifically mediating interactions between MPM cells and mesothelial cells.

\section{ACKNOWLEDGEMENTS}

We thank Keiko Kimura (Saiseikai Nakatsu Hospital, Osaka, Japan) for technical assistance. This work is supported by grants from the Ministry of Education, Culture, Sports, Science and Technology of Japan; and Kobayashi Magobe Memorial Medical Foundation.

1. Churg A, Cagle PT, Roggli VL. AFIP Atlas of Tumor Pathology Series 4: Tumors of the Serosal Membranes. ARP Press: Washington, DC, 2006.

2. Yan TD, Welch L, Black D, et al. A systematic review on the efficacy of cytoreductive surgery combined with perioperative intraperitoneal chemotherapy for diffuse malignancy peritoneal mesothelioma. Ann Oncol 2006;18:827-834.

3. Lee AY, Raz DJ, He B, et al. Update on the molecular biology of malignant mesothelioma. Cancer 2007;109:1454-1461.

4. Wakayama T, Ohashi K, Mizuno K, et al. Cloning and characterization of a novel mouse immunoglobulin superfamily gene expressed in early spermatogenic cells. Mol Reprod Dev 2001;60:158-164.

5. Kuramochi M, Fukuhara $H$, Nobukuni $T$, et al. TSLC1 is a tumorsuppressor gene in human non-small-cell lung cancer. Nat Genet 2001;27:427-430.

6. Biederer T, Sara Y, Mozhayeva M, et al. SynCAM, a synaptic adhesion molecule that drives synapse assembly. Science 2002;297:1525-1531.

7. Shingai $T$, Ikeda W, Kakunaga $S$, et al. Implications of nectin-like molecule-2/IGSF4/RA175/SgIGSF/TSLC1/SynCAM1 in cell-cell adhesion and transmembrane protein localization in epithelial cells. J Biol Chem 2003;278:35421-35427.

8. Wakayama $T$, Sai $Y$, Ito $A$, et al. Heterophilic binding of the adhesion molecules poliovirus receptor and immunoglobulin superfamily $4 \mathrm{~A}$ in the interaction between mouse spermatogenic and Sertoli cells. Biol Reprod 2007;76:1081-1090.

9. Ito A, Okada M, Uchino $K$, et al. Expression of the TSLC1 adhesion molecule in pulmonary epithelium and its down-regulation in pulmonary adenocarcinoma other than bronchioloalveolar carcinoma. Lab Invest 2003;83:1175-1183.

10. Ito A, Nishikawa Y, Ohnuma K, et al. SgIGSF is a novel biliary-epithelial cell adhesion molecule mediating duct/ductule development. Hepatology 2007;45:684-694.
11. Wakayama $\mathrm{T}$, Koami $\mathrm{H}$, Ariga $\mathrm{H}$, et al. Expression and functional characterization of the adhesion molecule spermatogenic immunoglobulin superfamily in the mouse testis. Biol Reprod 2003;68:1755-1763.

12. Ito A, Jippo T, Wakayama $T$, et al. SgIGSF: a new mast-cell adhesion molecule used for attachment to fibroblasts and transcriptionally regulated by MITF. Blood 2003;101:2601-2608.

13. Furuno $\mathrm{T}$, Ito $\mathrm{A}$, Koma $\mathrm{Y}$, et al. The spermatogenic lg superfamily/ synaptic cell adhesion molecule mast-cell adhesion molecule promotes interaction with nerves. J Immunol 2005;174:6934-6942.

14. Jippo $T$, Morii $E$, Ito $A$, et al. Effect of anatomical distribution of mast cells on their defense function against bacterial infections: demonstration using partially mast cell-deficient $\mathrm{tg} / \mathrm{tg}$ mice. J Exp Med 2003;197:1417-1425.

15. Watabe K, Ito A, Koma Y, et al. Distinct roles for the SgIGSF adhesion molecule and c-kit receptor tyrosine kinase in the interaction between mast cells and the mesentery. Biochem Biophys Res Commun 2004;324:782-788.

16. Ke $\mathrm{Y}$, Reddel RR, Gerwin $\mathrm{Bl}$, et al. Establishment of a human in vitro mesothelial cell model system for investigating mechanisms of asbestos-induced mesothelioma. Am J Pathol 1989;134:979-991.

17. Usami $N$, Fukui $T$, Kondo $M$, et al. Establishment and characterization of four malignant pleural mesothelioma cell lines from Japanese patients. Cancer Sci 2006;97:387-394.

18. Ohashi M, Aizawa S, Ooka $\mathrm{H}$, et al. A new human diploid cell strain, TIG-1, for the research on cellular aging. Exp Gerontol 1980;15: 121-133.

19. Nakamoto K, Ito A, Watabe $\mathrm{K}$, et al. Increased expression of a nucleolar Nop5/Sik family member in metastatic melanoma cells: evidence for its role in nucleolar sizing and function. Am J Pathol 2001;159: 1363-1374.

20. Mimura $T$, Ito A, Sakuma $T$, et al. Novel marker D2-40, combined with calretinin, CEA, and TTF-1: an optimal set of immunodiagnostic markers for pleural mesothelioma. Cancer 2007;109:933-938.

21. Ito $T$, Shimada $Y$, Hashimoto $Y$, et al. Involvement of TSLC1 in progression of esophageal squamous cell carcinoma. Cancer Res 2003;63:6320-6326.

22. Lung HL, Leung Cheung AK, Xie D, et al. TSLC1 Is a tumor suppressor gene associated with metastasis in nasopharyngeal carcinoma. Cancer Res 2006;66:9385-9392.

23. Dulbecco R, Allen R, Okada $\mathrm{S}$, et al. Functional changes of intermediate filaments in fibroblastic cells revealed by a monoclonal antibody. Proc Natl Acad Sci USA 1983;80:1915-1918.

24. Yabuta $\mathrm{N}$, Onda $\mathrm{H}$, Watanabe $\mathrm{M}$, et al. Isolation and characterization of the TIGA genes, whose transcripts are induced by growth arrest. Nucleic Acids Res 2006;34:4878-4892.

25. Mao X, Seidlitz E, Ghosh K, et al. The cytoplasmic domain is critical to the tumor suppressor activity of TSLC1 in non-small cell lung cancer. Cancer Res 2003;63:7979-7985.

26. Sasaki H, Nishikata I, Shiraga T, et al. Overexpression of a cell adhesion molecule, TSLC1, as a possible molecular marker for acute-type adult T-cell leukemia. Blood 2005;105:1204-1213.

27. Ito A, Koma $Y$, Watabe $K$, et al. Contribution of the SgIGSF adhesion molecule to survival of cultured mast cells in vivo. Biochem Biophys Res Commun 2004;319:200-206. 